\title{
Real-time dynamics of particle-hole excitations in Mott insulator-metal junctions.
}

\author{
Luis G. G. V. Dias da Silva, ${ }^{1,2}$ Khaled A. Al-Hassanieh, ${ }^{3}$ Adrian \\ E. Feiguin, ${ }^{4}$ Fernando A. Reboredo, ${ }^{2}$ and Elbio Dagotto ${ }^{1,2}$ \\ ${ }^{1}$ Department of Physics and Astronomy, University of Tennessee, Knoxville, Tennessee 37996 \\ ${ }^{2}$ Materials Science and Technology Division, Oak Ridge National Laboratory, Oak Ridge, Tennessee 37831 \\ ${ }^{3}$ Theoretical Division, Los Alamos National Laboratory, Los Alamos, New Mexico 87545 \\ ${ }^{4}$ Department of Physics and Astronomy, University of Wyoming, Laramie, Wyoming 82071, USA
}

(Dated: May 26, 2022)

\begin{abstract}
Charge excitations in Mott insulators (MIs) are distinct from their band-insulator counterparts and they can provide a mechanism for energy harvesting in solar cells based on strongly correlated electronic materials. In this paper, we study the real-time dynamics of holon-doublon pairs in a MI connected to metallic leads using the time-dependent density matrix renormalization group method. The transfer of charge across the MI-metal interface is controlled by both the electron-electron interaction strength within the MI and the voltage difference between the leads. We find an overall enhancement of the charge transfer as compared to the case of a (noninteracting) band insulatormetal interface with a similar band gap. Moreover, the propagation of holon-doublon excitations within the MI dynamically changes the spin-spin correlations, introducing time-dependent phase shifts in the spin structure factor.
\end{abstract}

PACS numbers: 71.10.Fd, 71.35.-y

\section{INTRODUCTION}

The dynamics of excitations in strongly correlated electronic materials (SCEMs) (Ref. 1) has been the subject of intense study in recent years. In a vast class of SCEMs, the ground state is a Mott insulator (MI), characterized by strong on-site repulsive interactions and a charge gap in the density of states. A paradigmatic model describing this behavior is the one-dimensional (1D) Hubbard model at half-filling, in which elementary charge excitations involve either doubly occupied (doublons) or empty (holons) electronic sites. A doublon-holon pair is thus a charge neutral excitation, which, depending on the relative strength of the on-site and nearest-site repulsion, can either form a bound state (a "Hubbard exciton") or remain decoupled. These excitonic states in Hubbard-type systems have attracted considerable attention involving both theoretical $2^{2 / 4}$ and experimenta $1^{5 / 7}$ investigations.

A particular framework where the behavior of doubly occupied site excitations has acquired considerable relevance is in experiments involving cold atomic fermionic mixtures in optical lattices ${ }^{8}$ While these experiments are performed under controlled conditions and allow for a substantial degree of tunability, the ability to properly probe and characterize these systems is limited. A recently proposed technique to probe the spectrum of a Mott insulating state consists of dynamically creating double occupancies by modulating the lattice depth with a time-dependent optical potential.$^{9}$ When the lattice depth exceeds the Mott gap, double occupancy becomes favorable and, by optical methods, it is possible to determine the appearance of the gapped mode. $\frac{10 \mid 11}{11}$ In addition, lattice-modulation spectroscopy has been proposed as a method to detect antiferromagnetic ordering and probe the nature (coherent or incoherent) of quasiparticle excitations. ${ }^{12}$ The decay mechanisms asso- ciated to the doublon excitations bring up fundamental questions about thermalization and non-equilibrium dynamics 13,15

In addition, technological applications may potentially arise from exploiting charge excitations in Mott insulators as a way to devise SCEM-based solar cells, which can offer structural and optical advantages over current devices made with semiconductor materials. Onedimensional Mott insulators (such as $\mathrm{Sr}_{2} \mathrm{CuO}_{3}$ ) are particularly known for strong nonlinear optical response effects $\frac{16[17}{16}$ that can be exploited in this context. The efficiency of SCEM-based solar cells will depend on several factors, including the performance of Mott insulatormetal junctions where the photocurrent will be generated. A crucial question is whether charge excitations in the MI will be able to properly transfer the charge into the metallic contacts, thus establishing a steadystate photocurrent.

Several questions arise in these regards: (i) Are the charge excitations long-lived? In other words, what are the effective decaying channels for the holon-doublon excitations into spin excitations inside the MI region? (ii) What are the effects of the dynamics of these excitations on the original correlations in the MI region? (iii) Can these charge excitations propagate across an interface with a non-correlated material?

Point (i) was addressed by some of us in Ref. 18, where the real-time dynamics of a holon-doublon pair was studied in a 1D Mott insulator. In that previous effort it was found that the decay to spin excitations in the underlying spin background is inefficient, and the pair is long-lived. The weak decay to spin-only excitations is particularly telling since the low-lying excitations for the 1D Hubbard model at half-filling carry spin (spinon) and charge (holons) quantum numbers separately and propagate with different characteristic velocities, as described 


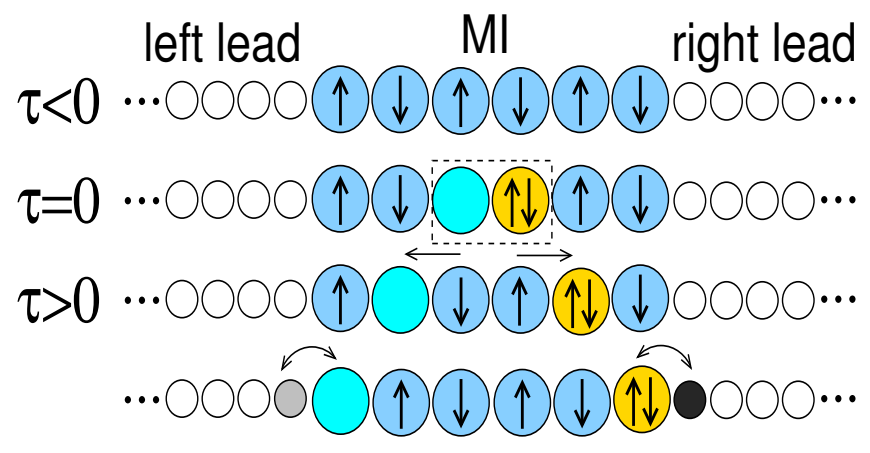

FIG. 1: Schematic representation of the MI+leads system studied here. At time $\tau=0$, a doublon-holon pair is created at the center of the MI region. This pair propagates and eventually reaches the boundary interfaces.

by the Tomonaga-Luttinger liquid picture. The ensuing spin-charge separation of these low-energy modes has been long studied and the real-time dynamics has been explored in recent efforts $[19120$

Points (ii) and (iii) will be addressed in this paper. Here, we study the charge and spin dynamics across a $\mathrm{MI} /$ metal junction, as doublon-holon excitations are created within the MI. A possible mechanism for such excitations arises from optical absorption at energies on the order of the Mott gap, producing exciton-like states. We study the time evolution of a localized doublon-holon pair initially created within the MI region. We consider a regime with weak nearest-neighbor electron-electron repulsion relative to the kinetic energy within the MI (or, equivalently, weak holon-doublon attraction), leading to a spatial dissociation of the doublon-holon pair, with both excitations eventually reaching the MI-metal boundaries.

The main results are summarized as follows. A strong confinement of the holon-doublon pairs within the MI region occurs for moderate to high values of the on-site interaction $U$. Nevertheless, charge transmission through the MI-metal boundary can be favored by properly adjusting the voltage difference between the metallic leads. These dynamical effects have a strong influence on the spin-spin correlations within the MI region, effectively reducing the power-law decaying antiferromagnetic (AFM) order and introducing phase shifts in the spin structure factor.

The paper is organized as follows. The model and the details of the calculation are described in Sec. III The main results are presented in Sec. III where we discuss the charge, double occupation, and spin-spin correlation dynamics (Sec. III A), as well as compare results against noninteracting cases (Sec. III B). The phase shifts in the spin structure factor are also discussed (Sec. IIIC). A summary of the main results is given in Sec. IV.

\section{MODEL AND METHODS}

The metal-MI-metal junction studied here is described by a 1D Hubbard model with on-site and nearestneighbor Coulomb interaction terms (representing the MI region) connected to noninteracting sites (representing the metallic leads). The Hamiltonian reads $H=$ $H_{\mathrm{MI}}+H_{\text {leads }}+H_{\text {coupling }}$, with:

$$
\begin{aligned}
H_{\mathrm{MI}}= & -t^{\prime \prime} \sum_{\sigma, i=N_{L}+1}^{N_{L}+N_{\mathrm{MI}}} c_{i \sigma}^{\dagger} c_{i+1 \sigma}+U\left(n_{i \uparrow}-\frac{1}{2}\right)\left(n_{i \downarrow}-\frac{1}{2}\right) \\
& +V\left(n_{i}-1\right)\left(n_{i+1}-1\right)+\text { h.c. } \\
H_{\text {leads }}= & \sum_{i \in R, L} \mu_{R, L} n_{i}-t \sum_{\sigma, i \in R, L} c_{i \sigma}^{\dagger} c_{i+1 \sigma}+\text { h.c. } \\
H_{\text {coupling }}= & -t^{\prime} \sum_{\sigma, i=N_{L} ; i=N_{L}+N_{\mathrm{MI}}}\left(c_{i \sigma}^{\dagger} c_{i+1 \sigma}+\text { h.c. }\right)
\end{aligned}
$$

where the sum in $H_{\text {coupling }}$ has only two terms $\left(i=N_{\mathrm{L}}\right.$ and $\left.i=N_{\mathrm{L}}+N_{\mathrm{MI}}\right)$. As usual, $c_{i \sigma}^{\dagger}\left(c_{i \sigma}\right)$ represents the creation (destruction) operator of an electron with spin projection $\sigma$ at site $i$.

In the model above, $H_{\mathrm{MI}}$ describes the central interacting region, with $U$ and $V$ being, respectively, the on-site and nearest-neighbor Coulomb repulsion. $t^{\prime \prime}$ is the hopping matrix element between sites in the MI region. The second term $\left(H_{\text {leads }}\right)$ represents the noninteracting leads, where $t$ is the tight-binding hopping amplitude (taken as the unit of energy hereafter) and $\mu_{\ell}$ is the on-site diagonal energy at the sites forming the lead $\ell$. Finally, $H_{\text {coupling }}$ describes the coupling between the leads and the MI involving a hopping amplitude $t^{\prime}$.

The total length of the $1 \mathrm{D}$ chain representing the entire system is $N_{\mathrm{L}}+N_{\mathrm{MI}}+N_{\mathrm{R}}$, where $N_{\mathrm{L}}, N_{\mathrm{MI}}$, and $N_{\mathrm{R}}$ correspond to the number of sites in the left lead, the Mott insulator, and the right lead, respectively (referred to as a " $N_{\mathrm{L}}-N_{\mathrm{MI}}-N_{\mathrm{R}}$ configuration"). The key parameters governing the dynamics are the on-site interaction $U$ within the MI and the on-site energy difference between the leads, given by $\Delta \mu \equiv\left(\mu_{\mathrm{L}}-\mu_{\mathrm{R}}\right) / 2$, which can be controlled by applying different voltages to the leads (for isolated leads in equilibrium at a fixed density, $\Delta \mu$ will be the chemical potential difference between the leads). In the following, we consider symmetric chains with $N_{\mathrm{L}}=N_{\mathrm{R}}=20$ and $N_{\mathrm{MI}}=10$ (for a total of 50 sites for the system) and assume a strong lead-MI tunneling amplitude $\left(t^{\prime}=t\right)$, which minimizes reflections at the interface due to a hopping matrix mismatch (although reflections due to the fact that $t^{\prime \prime} \neq t$ still play a role).

To investigate the dynamics of the excitations and tunneling into the metallic leads, we will focus on the regime of weakly bound doublon-holon pairs with negligible recombination probability. Following Ref. 18, these constraints can be met by choosing $U$ and $V$ such that $U / t^{\prime \prime} \gtrsim 8$ and $V / t^{\prime \prime} \lesssim 0.6$. Thus, here we select the values $t^{\prime \prime}=0.5 t, V=0.3 t$, and $U>4$ for our studies. 
As expressed before, all parameters with units of energy hereafter are given in units of the hopping $t$.

Figure 1 depicts the metal-MI-metal junction. The equilibrium ( $\tau<0$ ") ground-state $|\psi\rangle_{0}$ is obtained from static density-matrix renormalization group (DMRG) (Refs. 21/22) calculations. At time $\tau=0$, a doublonholon pair is suddenly created, by acting with holon $\left(h_{i}^{\dagger}\right)$ and doublon $\left(d_{i}^{\dagger}\right)$ creation operators on the state $|\psi\rangle_{0}$ :

$$
|\psi(\tau=0)\rangle=h_{p}^{\dagger} d_{p+1}^{\dagger}|\psi\rangle_{0}
$$

with $h_{i}^{\dagger} \equiv(1 / \sqrt{2}) \sum_{\sigma} c_{i \sigma}\left(1-n_{i \bar{\sigma}}\right)$ and $d_{i}^{\dagger} \equiv$ $(1 / \sqrt{2}) \sum_{\sigma} c_{i \sigma}^{\dagger} n_{i \bar{\sigma}}$.

The site where the holon is created is chosen as $p=$ $N_{\mathrm{L}}+N_{\mathrm{MI}} / 2$ so that the doublon-holon pair is initially localized at the center of the MI region. This choice, although not crucial, keeps the system symmetric under the application of a particle-hole transformation followed by a reflection through the middle bond.

The real time dynamics is obtained by time-evolving the original state using the time-dependent DMRG method $23 \mid 24$ and obtaining $|\psi(\tau)\rangle=e^{-i \hat{H} \tau}|\psi(0)\rangle$. We find that a Suzuki-Trotter decomposition with time steps $\delta \tau=0.05-0.1$ and keeping 200 states during the time evolution provides an adequate choice for time-evolved quantities up to times $\tau \sim 40$. Since the sudden creation of an exciton via Eq. 2 is restricted to only two sites in the original system, we find that, starting from a well converged initial state, increasing the number of states during the time evolution does not significantly alter the main results.

To probe the effects of the doublon-holon dynamics on the properties of the system, we calculate the expectation values of local operators $\hat{\mathcal{O}}_{i}$ at site $i$ at each time step. We define the change from the corresponding equilibrium value as $\delta \hat{\mathcal{O}}_{i}(\tau) \equiv\left\langle\hat{\mathcal{O}}_{i}\right\rangle(\tau)-\left\langle\hat{\mathcal{O}}_{i}\right\rangle_{\text {eq }}$, where $\langle\ldots\rangle$ indicates the expectation value using the time-evolved state $|\psi(\tau)\rangle$ at time $\tau$ while $\left\langle\hat{\mathcal{O}}_{i}\right\rangle_{\text {eq }}$ is the value calculated at equilibrium (" $\tau<0$ "). Typical site operators considered here are the on-site charge $n_{i}$ and double occupation $D_{i} \equiv d_{i}^{\dagger} d_{i}$.

We also define the "charge transfer" to the right metallic lead as

$$
\Delta n_{\mathrm{R}}(\tau, \Delta \mu) \equiv \sum_{i \in R}\left\langle n_{i}\right\rangle(\tau)-\left\langle n_{i}\right\rangle_{\mathrm{eq}}=\sum_{i \in R} \delta n_{i}(\tau),
$$

where the sum runs over sites in the right lead only. This quantity keeps track of the time-integrated charge that is transported into the right lead as a result of the creation of the holon-doublon excitation at $\tau=0$. A small charge transfer would indicate strong confinement of the doublon-holon pair within the MI region.

We should point out that, as defined in Eq. 2, the doublon is created to the "right" of the holon (i.e., a "left/right" holon-doublon pair), making the right lead the "doublon side" and thus justifying the choice of the right lead for the definition of the charge transfer in Eq.
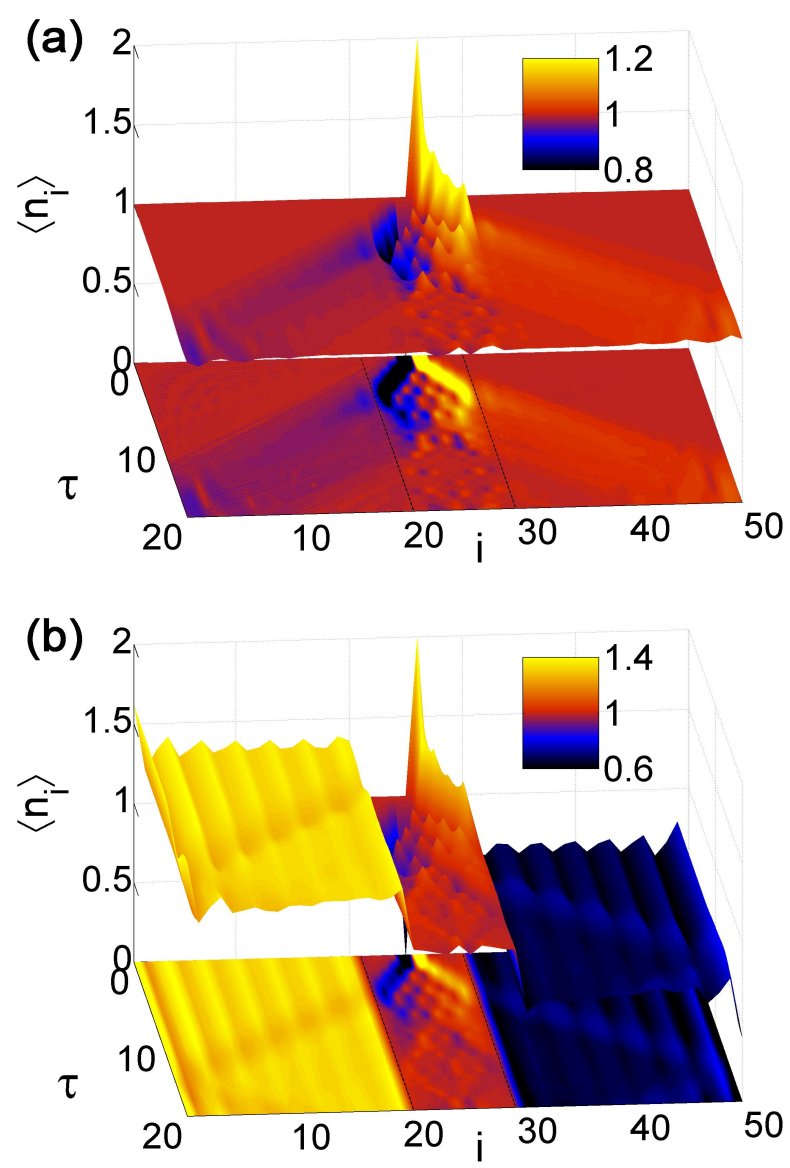

FIG. 2: Charge $\left\langle n_{i}\right\rangle$ at site $i$ versus time $\tau$ for a chain with $N=50$ sites (20-10-20 configuration), using $U=4$ and (a) $\Delta \mu=0$ and (b) $\Delta \mu=-1$.

3. This is only an arbitrary convention since the creation of "left/right" and "right/left" holon-doublon pairs by optical absorption should occur with equal probability. In fact, the effective total charge transfer to the right metallic lead should take into account the contribution from a "right/left" holon-doublon pair as well. Given the symmetries of the system described above, this contribution will be given by $-\Delta n_{\mathrm{R}}(\tau,-\Delta \mu)$ calculated for the left/right holon-doublon pair. Note that, once this contribution is taken into account, the net current is nonzero only for $\Delta \mu \neq 0$

Additionally, we investigate the spin-spin correlations away from equilibrium. We calculate the dynamical spin structure factor $S(q, \tau)$, defined as:

$$
S(q, \tau)=\frac{1}{N} \sum_{j, k \in \mathrm{MI}} e^{i(j-k) q}\left\langle S_{j}^{z} S_{k}^{z}\right\rangle(\tau),
$$

with $j, k$ spanning the interacting region $(j, k \in \mathrm{MI})$. As usual, a peak in $S(q)$ at $q=\pi$ signals quasi-longrange antiferromagnetic order, given in real space by $\left\langle S_{j}^{i} S_{j+k}^{z}\right\rangle \sim(-1)^{k} / r^{\alpha}$ with $\alpha$ being the critical exponent on the order parameter. 


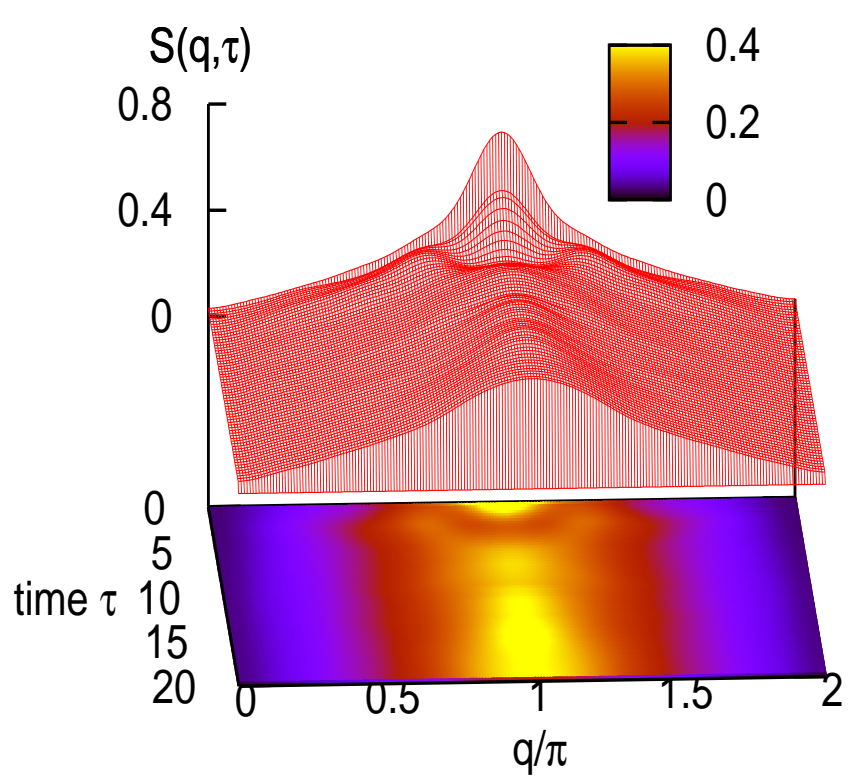

FIG. 3: Spin structure factor $S(q, \tau)$ in the MI region for $U=4$ and $\Delta \mu=0$.

\section{RESULTS}

\section{A. MI-leads charge transfer}

The typical dynamical behavior of the local charge $\left\langle n_{i}\right\rangle(\tau)$ is depicted in Fig. 2 a for $U=4$ and $\Delta \mu=0$. At $\tau<0$, the ground state of the system is particle-hole symmetric, with $\left\langle n_{i}\right\rangle=1$ for all $i$. At $\tau=0$, the doublonholon pair is created, making $\left\langle n_{p}\right\rangle=0,\left\langle n_{p+1}\right\rangle=2$, and $\left\langle D_{p}\right\rangle=0,\left\langle D_{p+1}\right\rangle=1$ (note that, while the total charge $\sum_{i} n_{i}$ is conserved, $\sum_{i} D_{i}$ is not constant). Due to the on-site repulsion in the MI, these charge excitations move in opposite directions as a function of $\tau$, eventually reaching the MI-metal interfaces at a time scale $\tau \sim \tau_{r}$ (for the parameters in Fig. 2, $\tau_{r} \approx 5.5$ ).

For $\Delta \mu \neq 0$, the charge is expected to be non-uniformly distributed, with clear charge "plateaus" in each of the three regions. This is clearly depicted in Fig. 2-b, with $\mu_{\mathrm{R}}>\mu_{\mathrm{L}}(\Delta \mu<0)$. In this case, the equilibrium charge distribution of the system is such that $\left\langle n_{i \in L}\right\rangle>1$, and $\left\langle n_{i \in R}\right\rangle<1$ while $\left\langle n_{i \in \mathrm{MI}}\right\rangle \sim 1$ in the MI region. As the doublon excitation approaches the MI-leads boundary, it is then partially transmitted to the right lead while, by symmetry, the holon excitation is partially transmitted to the left lead.

An intuitive picture for such a charge transmission/reflection can be formulated in terms of the singleparticle density of states. The energy cost for the formation of the doublon is on the order of the Mott gap, $\Delta_{\mathrm{MI}}$. Therefore, from purely energetic considerations, one expects an enhanced charge transport if there are enough states available in the leads at energies on the
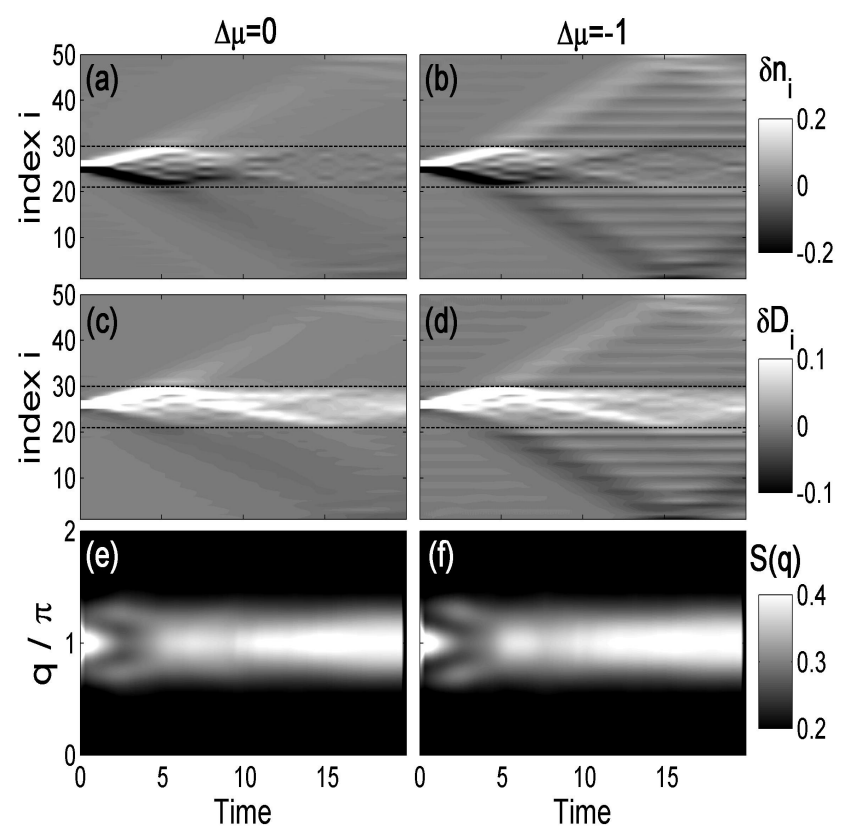

FIG. 4: Nonequilibrium charge difference $\delta n_{i}$ (a,b), double occupation $\delta D_{i}$ on each site (c,d), and spin structure factor $S(q, \tau)$ in the MI region (e,f) versus time. The results are for $U=4$ with different values of $\Delta \mu: \Delta \mu=0$ (a,c,e) and $\Delta \mu=-1(\mathrm{~b}, \mathrm{~d}, \mathrm{f})$. Note the pronounced charge transfer for $\Delta \mu=-1$.

order of $\pm \Delta_{\mathrm{MI}} / 2$ relatively to the Fermi energy. Static DMRG calculations for the equilibrium density of states (not shown) give Mott gap values $\Delta_{\mathrm{MI}} \approx 2$ for $U=4$ and $\Delta_{\mathrm{MI}} \approx 8$ for $U=10$, which is on the order or larger than the typical half bandwidth $\sim 2 t$ of the noninteracting chain. Therefore, for a fixed $\Delta \mu$, one expects a decreasing charge transfer with increasing $U$ as there are fewer states in the leads available at energies $\sim \pm \Delta_{\mathrm{MI}}$. On the other hand, a nonzero $\Delta \mu$ can increase the charge transfer by allowing a "matching" of states available for $|\Delta \mu| \sim \Delta_{\mathrm{MI}} / 2$.

In fact, the reflection at the boundary can be reduced by changing $\Delta \mu$, as shown in Fig. 2 -b. In this case, the Fermi energy in the leads is aligned with the band, creating states available for the excitation to "leak" into the leads.

This simplified picture, however, does not take into account the existing correlation effects within the Mott insulator region. Additional insight on the effect of the doublon-holon dynamics can be provided by the spin-spin correlations within the MI region, via the spin structure factor. Fig. 3 shows the time evolution for $S(q, \tau)$ calculated within the interacting (MI) region for $U=4$. In equilibrium (" $\tau<0$ ", shown as the first curve), a pronounced peak is observed at $q=\pi$ in both cases, signaling AFM correlations.

At intermediate times, this peak acquires "shoulders" which become more prominent around $\tau \sim \tau_{r} / 2$, which coincides with the time scale for which the doublon-holon 

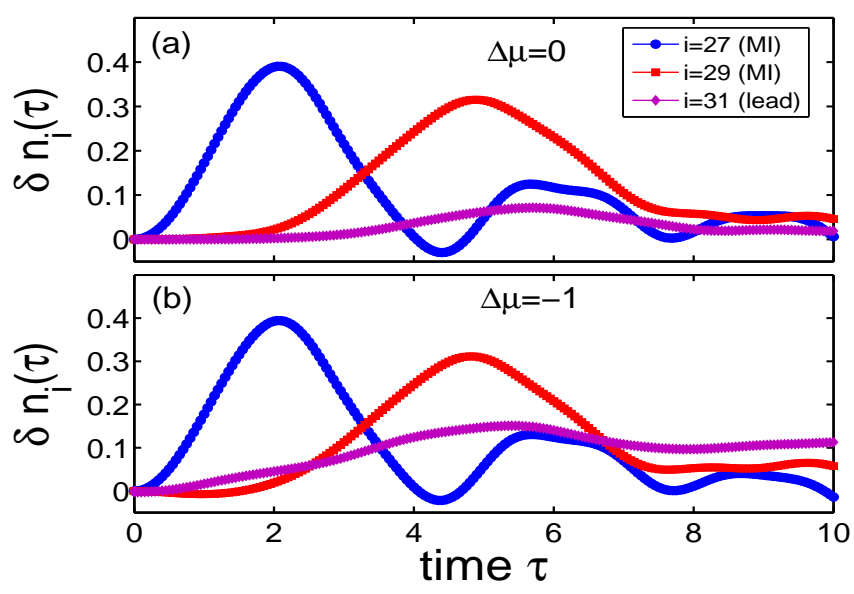

FIG. 5: Charge difference $\delta n_{i}(\tau)$ on sites near the MI-metal boundary as a function of time for $U=4$ and (a) $\Delta \mu=0$ and (b) $\Delta \mu=-1$.

pair is spatially separated but has not yet reached the boundary. For longer times, the peak is broadened, indicating a weaker AFM order within the MI region. The shoulders in $S(q, \tau)$ are consistent with the formation of anti-phase domain walls in the MI region due to the spatial separation of the doublon and the holon, as we discuss further in Sec. IIIC. The fact that these features in the spin structure factor closely track the charge dynamics indicates that charge excitations (rather than collective spin excitations) are the dominant decay channel for the doublon-holon pair, in accordance to previous findings! 18

Figure 4 presents a visual summary of the dynamics of the charge, double occupation, and spin-spin correlations for $U=4$ and different values of $\Delta \mu$. Figs. 4 a-b show $\delta n_{i}(\tau)$ at all sites for $\Delta \mu=0$ and $\Delta \mu=-1$, respectively. An enhanced charge transmission into the leads is clearly seen for $\Delta \mu=-1$, consistent with the arguments given above. This additional charge is an effect of the doublon excitation tunneling into the leads. This is confirmed by analyzing the change in double occupation from equilibrium $\delta D_{i}(\tau)$ (Fig. $4 \mathrm{c}-\mathrm{d}$ ). Note that the doublon is "long lived", as evidenced by the dynamics of the double occupation within the MI region as it reflects off the boundary. This is a consequence of the weak decay of the doublon for large $U / t^{\prime \prime}$, as discussed in Ref. 18.

Additionally, there are interesting effects in the spinspin correlation, as shown in the $S(q, \tau)$ plots in Figs. 4 e-f. A clear splitting of the peak occurs as the doublon and holon excitations become separated within the MI, also seen in Fig. 3. More interestingly is the fact that this feature is enhanced for $\Delta \mu<0$ (Fig. $4 \mathrm{f}$ ), a consequence of having a stronger charge tunneling between the MI and the leads (i.e. stronger "doping", as discussed in Sec. III C.

A closer look on the charge dynamics at the interface is presented in Fig. 5, which depicts the behavior of the added charge $\delta n_{i}(\tau)$ at sites on both sides of the inter-
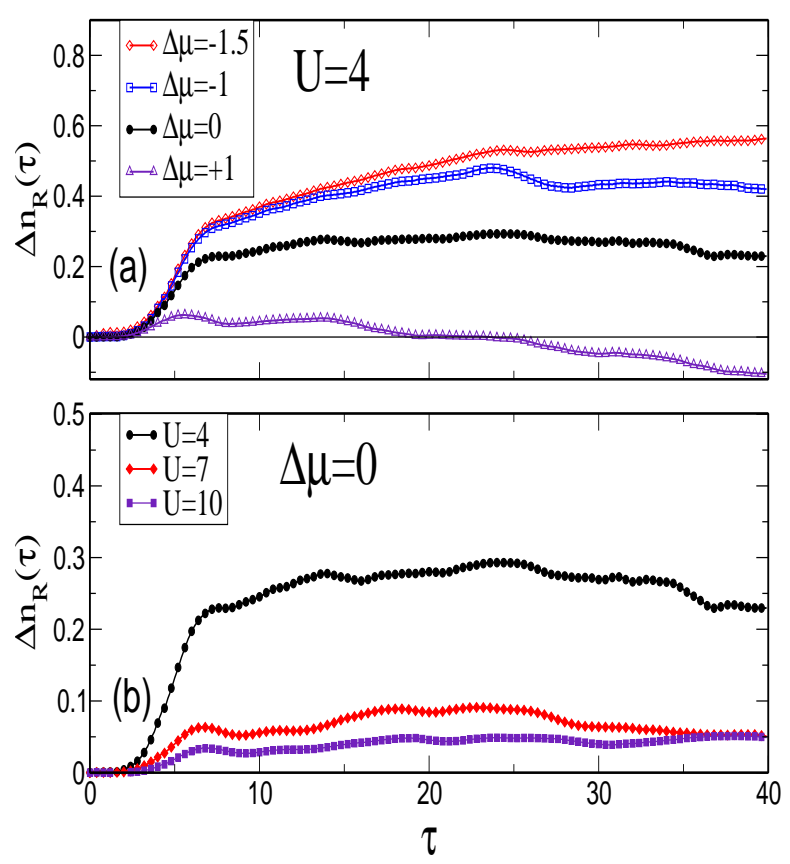

FIG. 6: Charge transfer (Eq. 3) for (a) $U=4$ and different values of $\Delta \mu$ and (b) $\Delta \mu=0$ and different values of $U$, with $t^{\prime \prime}=0.5$ in all cases.

face. A weak odd-even modulation is present so only odd-numbered sites are shown for a meaningful comparison. The amplitude of the charge excitation decays as the "charge front" approaches the boundary. In addition, a reflection at the interface takes place for $\tau \sim \tau_{r}=5.5$ in both cases.

We can estimate the MI-metal charge reflection by comparing the maximum amplitudes of $\delta n_{i}(\tau)$ at sites on each side of the interface. For $\Delta \mu=0$ (Fig. 5 . a), the maximum in $\delta n_{i}$ drops from 0.315 for $i=29$ (within the MI) to 0.071 for $i=31$ (in the leads), giving $R \equiv\left(\max \delta n_{29}-\max \delta n_{31}\right) / \max \delta n_{29}=0.7737$. The relative drop is smaller for $\Delta \mu=-1$ (Fig. 5-b), with $R \approx 0.51$.

For longer times, further scattering of the doublon and holon excitations off the MI-metal interface result in additional charge tunneling into the leads. The integrated charge transferred to the right lead $\left(\Delta n_{\mathrm{R}}\right.$, defined in Eq. 3) is plotted in Fig. 67a for different values of $\Delta \mu$. For longer times, $\Delta n_{\mathrm{R}}(\tau)$ reaches an approximate plateau, indicating a steady state of the charge in the lead (for $\Delta \mu=-1.5$ the plateau has not been fully reached on the maximum time used in the calculations). As expected from our previous results, the height of the plateau is larger for negative values of $\Delta \mu$. For a stronger interaction $U$ (larger $\Delta_{\mathrm{MI}}$ ), the charge transfer is strongly suppressed, as shown in Fig. 6. b.

These results indicate that the stronger the interaction $U$ in the MI region, the more confined the holon-doublon pair becomes. This is clearly seen in Figs. 7(a,b) and $(\mathrm{c}, \mathrm{d})$, which depicts contour plots of $\delta n_{i}(\tau)$ and $\delta D_{i}(\tau)$ for $U=4$ and $U=10$, respectively. For $U=10$, most of the charge remains confined in the MI region, reflect- 

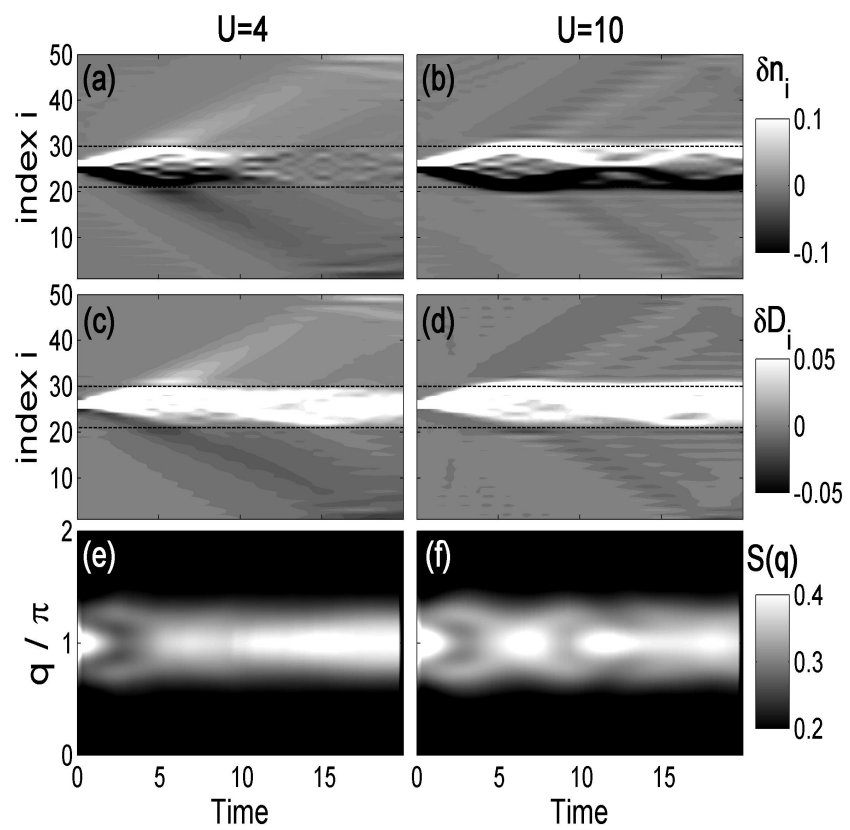

FIG. 7: Nonequilibrium charge $\delta n_{i}(\tau)(\mathrm{a}, \mathrm{b})$ and double occupation $\delta D_{i}(\tau)(\mathrm{c}, \mathrm{d})$ on each site as a function of time for $U=4(\mathrm{a}, \mathrm{c})$ and $U=10(\mathrm{~b}, \mathrm{~d})$. Panels (e,f) show the corresponding spin structure factor $S(q, \tau)$ in the MI region.

ing off the interface in a periodic pattern. Interestingly, the long-lived holon and doublon excitations also "repel" each other, as seen for $\tau \sim 2 \tau_{r}$.

Not surprisingly, increasing the Mott gap has a pronounced effect on the spin structure factor $S(q, \tau)$. Figs. $7 \mathrm{e}$ and $7 \mathrm{f}$ show $S(q, \tau)$ for $U=4$ and $U=10$, respectively. For $U=4$, the three-peak structure ("shoulders") disappear for $\tau \gtrsim \tau_{r}$, where a broad peak takes over. This is about the time scale for which the charge/spin fronts reach the boundary (Fig. 7fb). For $U=10$, the shoulders reappear at later times, consistent with the "beating" in the doublon/holon pair as they reflect back and forth within the MI region and with each other. This is coupled with a much stronger confinement of the holondoublon pair within the MI region, as seen in Figs. 7 bb.

\section{B. Comparison with the non-interacting case}

An important question is how the results for the MIleads charge transfer compare to a case where no electronelectron interactions are present in the central region. To address this question, we present results for two distinct cases: (i) a full "metallic" system, i.e., $U=V=0$ and $t^{\prime \prime}=0.5$ in the $H_{\mathrm{MI}}$ term in Eq. 1 and (ii) a "band insulator" region, in which $H_{\mathrm{MI}}$ is replaced by

$$
H_{\mathrm{BI}}=-t^{\prime \prime} \sum_{\sigma, i=N_{L}+1}^{N_{L}+N_{\mathrm{MI}}}\left(1+\delta(-1)^{i}\right) c_{i \sigma}^{\dagger} c_{i+1 \sigma}+\text { h.c. }
$$
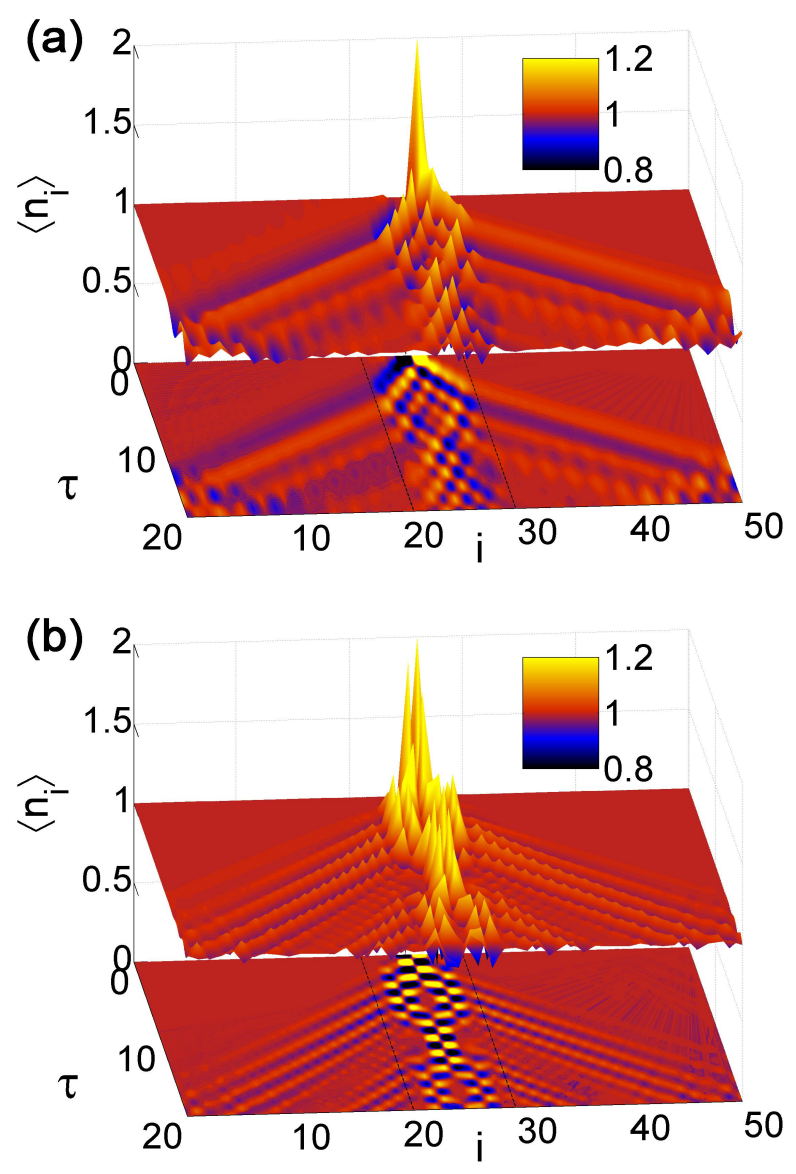

FIG. 8: Charge $\left\langle n_{i}\right\rangle$ on site i versus time $\tau$ for $N=50$ sites, a non-interacting central region $(U=0)$, and $\Delta \mu=0$. (a) is using a Hamiltonian as in Eq. 1 with $t^{\prime \prime}=0.5$ while in (b) $H_{\mathrm{BI}}$ replaces $H_{\mathrm{MI}}$ and $t^{\prime \prime}=1, \delta=0.6$ (band insulator).

in the Hamiltonian. Notice that $H_{\mathrm{BI}}$ represents a Peierls chain, which has a charge gap for $\delta \neq 0$ and the gap size will depend on $t^{\prime \prime}$ and $\delta$. In the following, we choose $t^{\prime \prime}=1$ and $\delta=0.6$ so that the band gap is nearly the same as the Mott gap for the case shown in Fig. 2 a $\left(U=4, V=0.3\right.$, and $\left.t^{\prime \prime}=0.5\right)$.

Figures 8 a and $\mathrm{b}$ show the occupation $\left\langle n_{i}\right\rangle$ on the chain sites for the fully metallic and band insulator cases, respectively. The first case corresponds to the noninteracting limit of the results presented in the previous section, with a fully metallic chain. There are still strong reflections at the boundary due to the hopping mismatch $\left(t^{\prime \prime} \neq t\right)$. More importantly, the holon-doublon charge excitations produce Friedel-type oscillations along the chain, forming a clear charge interference pattern over time (see contour plot in Fig. 8-a).

For the case where the central region is a band insulator, charge oscillations also occur, leading to a "checkerboard" pattern of alternating positive and negative charges in the central sites (Fig. 8 b). In this case, propagation of a "charge wavefront" is clearly suppressed as compared to the fully metallic case. 


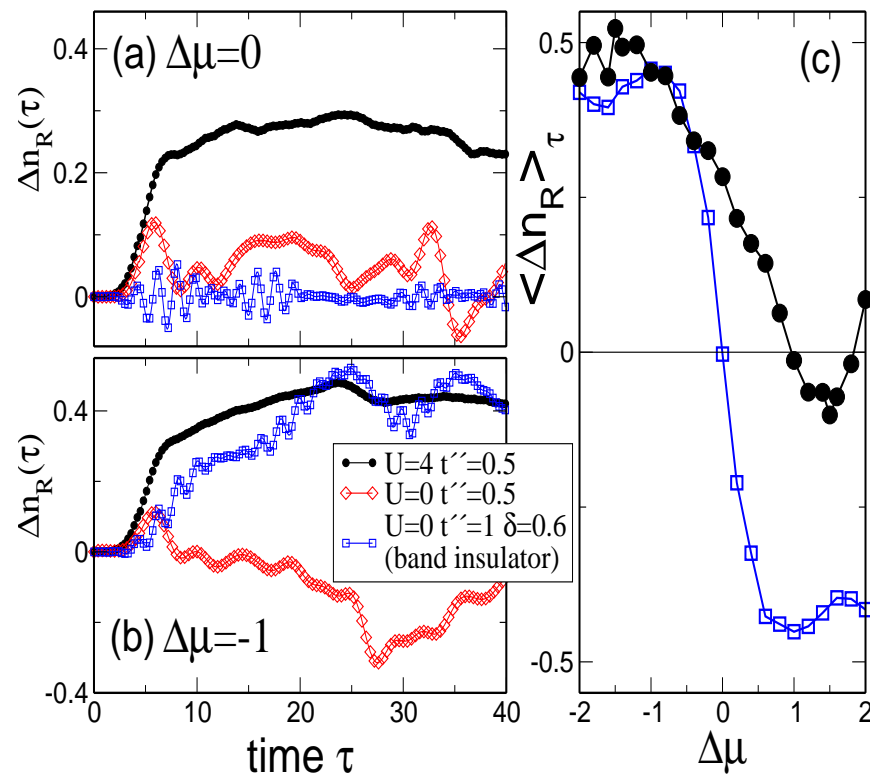

FIG. 9: Charge transfer comparison between the cases of interacting $(U=4)$ and noninteracting $(U=0)$ central regions. Results are also shown for a band insulator (Peierls chain) with a gap very similar to that of the Mott insulator with $U=4$. (a,b) $\Delta n_{R}(\tau)$ versus $\tau$ for $\Delta \mu=0$ and $\Delta \mu=-1$, respectively. (c) Time-averaged (steady-state) charge transfer $\left\langle\Delta n_{R}\right\rangle_{\tau}$ versus $\Delta \mu$ for the $\mathrm{MI}$ and BI cases.

The charge transfer to the right lead in both situations is clearly distinct from the interacting case. Figs. 9-a,b show a comparison of the charge transfer at $U=4$ (same as depicted in Fig. 60 with results at $U=0$ for $\Delta \mu=0$ and $\Delta \mu=-1$, respectively.

The charge transfer is very limited for $\Delta \mu=0$ in the noninteracting cases, having essentially a zero timeaverage for the band insulator. By contrast, the case $U=4$ shows a positive transfer even for $\Delta \mu=0$ due to the on-site repulsion within the central region. Note that the charge gap in the central region is nearly the same for both the Mott and band insulator cases, while the reflection at the boundary is much more accentuated for the latter. This highlights the role of the electronelectron interactions in the additional charge transfer for the $U=4$ case. For $\Delta \mu=-1$ (Fig. 9-b) the charge transfer is improved in the noninteracting cases, as expected. Overall, the charge transfer is still more effective in the case where the central region is a Mott insulator, as compared to the noninteracting cases.

In fact, this holds for a wide range of values of $\Delta \mu$, as it can be seen in Fig. 9 $\mathrm{c}$, where a time-averaged charge transfer $\left\langle\Delta n_{R}\right\rangle_{\tau}$ (calculated by taking averages of $\Delta n_{R}(\tau)$ over a time interval $\Delta \tau \sim 10$ at longer times, away from the initial transient) is plotted as a function of $\Delta \mu$. In the MI case, $\left\langle\Delta n_{R}\right\rangle_{\tau}$ shows a broad peak, over a wide range of $\Delta \mu$ (larger than the typical metallic halfbandwidth, $\sim 2$ in the units used), showing a positive charge transfer to the right lead even for positive values of $\Delta \mu$ (i.e., $\mu_{R}<\mu_{L}$ ). This is in sharp contrast with the

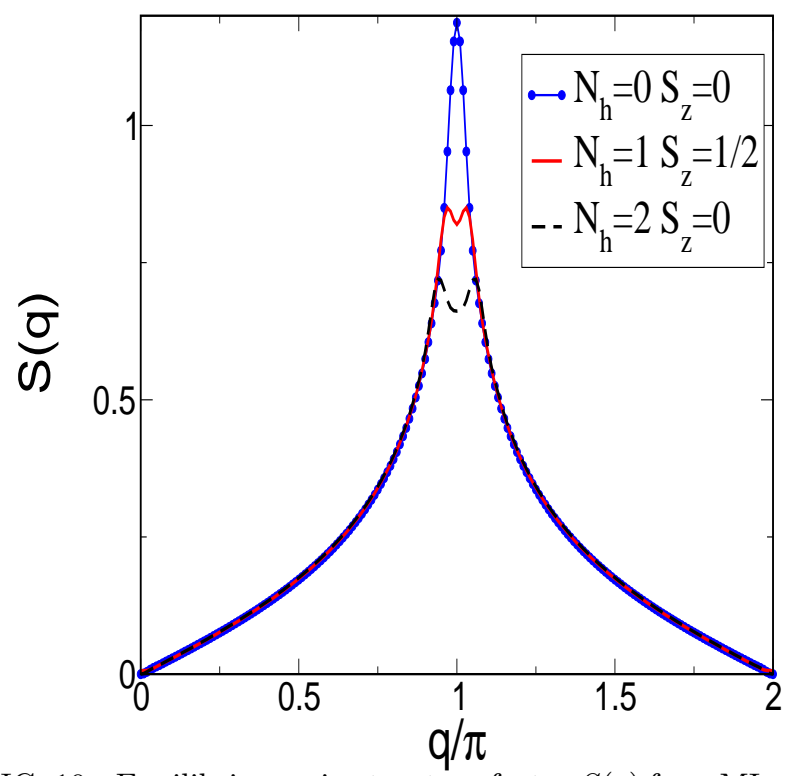

FIG. 10: Equilibrium spin structure factor $S(q)$ for a MI-only chain with $N$ sites (no leads) calculated for states with charge $N-N_{\mathrm{h}}$ ( $N_{\mathrm{h}}$ is the number of holes) and spin $S_{z}$. Parameters are $U=4$ and $t^{\prime \prime}=0.1$.

band insulator case, for which $\left\langle\Delta n_{R}\right\rangle_{\tau}<0$ for $\mu_{R}<\mu_{L}$. Notice that, for $\Delta \mu=0,\left\langle\Delta n_{R}\right\rangle_{\tau}>0$ in the MI case while $\left\langle\Delta n_{R}\right\rangle_{\tau} \approx 0$ in the BI case (Fig. 9-c). The latter is an expected result: in a noninteracting system with an initially uniform charge distribution, an electron-hole excitation will produce charge oscillations about the initial charge value (see Fig. 8) but, on average, no charge transfer takes place.

\section{Phase shifts in $\mathbf{S}(\mathbf{q})$}

To understand the three-peak feature in the spin structure factor (e.g., Fig. 3), we have calculated $S(q)$ for a Hubbard chain with $N_{\mathrm{L}}=N_{\mathrm{R}}=0$ and $N_{\mathrm{MI}}=40$ sites using static DMRG. We performed several DMRG calculations targeting states with different number of electrons $N$ and total spin projection $S_{z}$. More specifically, we considered half-filling $\left(N=N_{\mathrm{MI}}\right)$ and hole-doped ( $N=N_{\mathrm{MI}}-N_{\mathrm{h}}$ where $N_{\mathrm{h}}$ is the number of holes) cases, with $S_{z}=0,1 / 2$, and calculated the corresponding $S(q)$ for the different situations.

Results are presented in Fig. 10 . For $N_{\mathrm{h}}=0$ and $S_{z}=0$, the state corresponds to the antiferromagnetically ordered ground-state of the system at half-filling, with a peak at $q=\pi$. We then targeted states with different values of $N_{\mathrm{h}}$ and $S_{z}$. This corresponds to calculating the ground-state of a system in which holes and/or spin flips are added. For $N_{\mathrm{h}}=1, S_{z}=1 / 2$ (one hole) and $N_{\mathrm{h}}=2$, $S_{z}=0$ (two holes) a double peak structure appears.

This is reasonable since holons will introduce antiphase domain walls (ADW) (i.e. " $\pi$-shifts") in the AFM order and peaks at $\pi \pm \pi \nu_{h}$ are expected (where $\nu_{h}$ is the hole density). As spin-flips are added (for instance, 
$\left.N_{\mathrm{h}}=1, S_{z}=3 / 2\right)$ the double-peak becomes four peaks (not shown). In light of these static calculations, the appearance of "shoulders" in $S(q, \tau)$ for $\tau<\tau_{r}$ (where $\tau_{r}$ is the time it takes for the excitations to reach the boundaries) can be accounted for as follows.

The creation of the holon/doublon pairs at $\tau=0$ effectively removes two magnetic moments from the MI region, making $S_{p(p+1)}^{z}=0$ at the holon (doublon) site. This explains the decrease in the area under the $S(q, \tau)$ curve at $\tau=0$ as $\int S(q, \tau) d q \propto \sum_{i \in \mathrm{MI}}\left\langle\left(S_{i}^{z}\right)^{2}\right\rangle(\tau)$. For small $\tau, S(q, \tau)$ still retains a single-peak structure initially since holon and doublon are on adjacent sites and the corresponding phase shifts from ADWs cancel out.

For $\tau<\tau_{r}$, the MI region can be divided into two parts with magnetically distinct characteristics: (i) an "undoped" region where neither the doublon or holon excitations have arrived and which still retains AFM order and (ii) a "doped" region, which has been already "covered" by either the doublon or the holon excitations. Part (i) contributes to a peak at $q=\pi$ in $S(q, \tau)$ while part (ii) contributes to the two shoulders, as we expect from the static calculations shown in Fig. 10 with $N_{\mathrm{h}}=2$.

For $\tau \sim \tau_{r}$, the excitation (and the corresponding ADW) reaches the boundaries, making the phase shifts to cancel out again, suppressing the side peaks. As the holon and doublon reflect off the boundaries, the side peaks reappear up to time scales of order $\tau \sim 2 \tau_{r}$. At this time scale, doublon and holon excitations are again at nearest-neighbor sites and the respective ADW phaseshifts cancel once again, leading to a single-peak structure. The process then repeats up to time scales on the order of the doublon decay time. Thus, this cycle is observed more clearly for larger values of $U$ (e.g., Fig. $7 \mathrm{ff}$ ) for which the doublon decay time is large enough. Notice that $S\left(q, \tau \sim 2 \tau_{r}\right)$, although featuring a single peak at $q=\pi$, has a much smaller area than $S(q, \tau=0)$, indicating that the propagation of the holon-doublon pair significantly modifies the AFM correlations.

\section{SUMMARY}

In summary, we have studied the real-time propagation of doublon-holon excitations in a Mott insulator (MI) connected to metallic leads. We analyze the dynamics of charge, double occupation, and spin-spin correlations within the MI, as well as the MI-leads charge transfer.

Our results indicate that the sharp change in the Hamiltonian at the MI-metal interface hinders the charge transfer, suggesting that metals that closely resemble the structure of the MI (Ref. 16) would be required as charge collectors instead of standard doped semiconductors. More specifically, we find that the charge transfer across the MI-metal boundary is quite sensitive to microscopic parameters in the MI region, particularly the onsite interaction $U$. While $U$ needs to be sufficiently large so that the doublon decay-time is larger than the typical time scale it takes to reach the boundary, doublon-holon tunneling into the leads is suppressed for very large values of $U$.

We believe two factors contribute to these results: (i) the increase in the Mott gap and (ii) the fact that, for large $U$, the nature of the doublon and holon excitations within the MI becomes more different than standard electron and hole excitations in the noninteracting case. We have tested these hypothesis by comparing configurations with either a Mott insulator or a (noninteracting) band insulator in the central region: they show clear differences in the charge transfer.

The repulsive interaction within the Mott insulator region favors the transfer of the excess charge into the metallic leads even at $\Delta \mu=0$. This is in sharp contrast with a noninteracting band insulator connected to leads with the same band-gap for which the net charge transfer is essentially zero at zero voltage difference.

Noninteracting and interacting cases show clearly distinct charge dynamics after the excitation. In the former case, Friedel-type charge oscillations in the central region are prominent at small times, while they are suppressed in the interacting case and a clear spatial separation between hole-like and particle-like excitations occurs.

Moreover, the propagation of holons and doublons within the MI region dynamically alters the AFM spinspin correlations. In particular, extra " $\pi$ shifts" appear in the spin correlation functions as doublons and holons are spatially separated. We believe these qualitative findings will help on the prospect of making future solar cell devices using strongly correlated materials.

An interesting aspect that remains open is the effect of a finite temperature in our results, a difficult problem considering that the numerical study of transport in correlated systems at finite temperatures remains a very challenging subject. The energy scales of spin and charge excitations in 1D Mott insulators can be quite different $\left(\sim t^{\prime \prime}\right.$ for gapped charge excitations and $\sim\left(t^{\prime \prime}\right)^{2} / U$ for gapless spin excitations) and finite temperatures can affect each of these channels differently ${ }^{25 \mid 26}$ In our case, however, since the doublon-holon pairs decay very weakly into spinon modes and the dynamics is governed by charge excitations, we expect the results to hold as long as temperatures are small compared to the charge gap.

\section{Acknowledgments}

We acknowledge motivating discussions with Ivan Gonzalez, Fabian Heidrich-Meisner, Hiro Onishi, and Satoshi Okamoto. Research sponsored by the Division of Materials Sciences and Engineering, Office of Basic Energy Sciences, U.S. Department of Energy. Computational support was provided by the National Energy Research Scientific Computing Center (NERSC). Work at LANL was carried out under the auspices of the NNSA of the U.S. DOE under Contract No. DE-AC52-06NA25396. LGGVDS and ED acknowledge support from the National Science Foundation via grant DMR-0706020. 
1 Y. Tokura and N. Nagaosa, Science 288, 462 (2000).

2 W. Barford, Phys. Rev. B 65, 205118 (2002).

${ }^{3}$ E. Jeckelmann, Phys. Rev. B 67, 075106 (2003).

${ }^{4}$ H. Matsueda, T. Tohyama, and S. Maekawa, Phys. Rev. B 71, 153106 (2005).

${ }^{5}$ K. W. Kim, G. D. Gu, C. C. Homes, and T. W. Noh, Phys. Rev. Lett. 101, 177404 (2008).

6 A. Gössling, R. Schmitz, H. Roth, M. W. Haverkort, T. Lorenz, J. A. Mydosh, E. Müller-Hartmann, and M. Grüninger, Phys. Rev. B 78, 075122 (2008).

7 Y. Matiks, P. Horsch, R. K. Kremer, B. Keimer, and A. V. Boris, Phys. Rev. Lett. 103, 187401 (2009).

8 I. Bloch, J. Dalibard, and W. Zwerger, Rev. Mod. Phys. 80, 885 (2008).

9 C. Kollath, A. Iucci, T. Giamarchi, W. Hofstetter, and U. Schollwöck, Phys. Rev. Lett. 97, 050402 (2006).

10 R. Joerdens, N. Strohmaier, K. Guenther, H. Moritz, and T. Esslinger, Nature 455, 204 (2008).

11 S. D. Huber and A. Rüegg, Phys. Rev. Lett. 102, 065301 (2009).

12 R. Sensarma, D. Pekker, M. D. Lukin, and E. Demler, Phys. Rev. Lett. 103, 035303 (2009).

13 A. Rosch, D. Rasch, B. Binz, and M. Vojta, Phys. Rev. Lett. 101, 265301 (2008).

14 F. Heidrich-Meisner, S. R. Manmana, M. Rigol, A. Muramatsu, A. E. Feiguin, and E. Dagotto, Phys. Rev. A 80,
041603(R) (2009).

15 N. Strohmaier, D. Greif, R. Jordens, L. Tarruell, H. Moritz, T. Esslinger, R. Sensarma, D. Pekker, E. Altman, and E. Demler, Phys. Rev. Lett. 104, 080401 (2010).

16 H. Kishida, H. Matsuzaki, H. Okamoto, T. Manabe, M. Yamashita, Y. Taguchi, and Y. Tokura, Nature (London) 405, 929 (2000).

17 H. Kishida, M. Ono, K. Miura, H. Okamoto, M. Izumi, T. Manako, M. Kawasaki, Y. Taguchi, Y. Tokura, T. Tohyama, et al., Phys. Rev. Lett. 87, 177401 (2001).

18 K. A. Al-Hassanieh, F. A. Reboredo, A. E. Feiguin, I. González, and E. Dagotto, Phys. Rev. Lett. 100, 166403 (2008).

19 C. Kollath, U. Schollwöck, and W. Zwerger, Phys. Rev. Lett. 95, 176401 (2005).

20 T. Ulbricht and P. Schmitteckert, Europhys. Lett. 86, 57006 (2009).

21 U. Schollwock, Rev. Mod. Phys. 77, 259 (2005).

${ }^{22}$ K. A. Hallberg, Adv. Phys. 55, 477 (2006).

23 S. R. White and A. E. Feiguin, Phys. Rev. Lett. 93, 076401 (2004).

24 A. J. Daley, C. Kollath, U. Schollwöck, and G. Vidal, J. Stat. Mech.: Theory Exp. 2004, P04005 (2004).

${ }^{25}$ G. A. Fiete, Rev. Mod. Phys. 79, 801 (2007).

26 B. I. Halperin, J. Appl. Phys. 101, 081601 (2007). 